Article

\title{
Norhierridin B, a New Hierridin B-Based \\ Hydroquinone with Improved Antiproliferative Activity
}

\author{
Pedro Brandão $\left.{ }^{1,+} \mathbb{(}\right)$, Joana Moreira ${ }^{1,2,{ }^{\dagger}}$, Joana Almeida ${ }^{3} \mathbb{C}$, Nair Nazareth $^{3}$, \\ Ivo E. Sampaio-Dias ${ }^{(\mathbb{D}}$, Vitor Vasconcelos ${ }^{2,5}$ (D), Rosário Martins ${ }^{2,6}$, Pedro Leão ${ }^{2}$, \\ Madalena Pinto ${ }^{1,2}$, Lucília Saraíva ${ }^{3, *(D)}$ and Honorina Cidade ${ }^{1,2, *(D)}$ \\ 1 Laboratory of Organic and Pharmaceutical Chemistry, Department of Chemical Sciences, \\ Faculty of Pharmacy, University of Porto, Rua de Jorge Viterbo Ferreira 228, 4050-313 Porto, Portugal; \\ pedrocgbrandao@gmail.com (P.B.); joana.m26@hotmail.com (J.M.); madalena@ff.up.pt (M.P.) \\ 2 CIIMAR/CIMAR, Interdisciplinary Centre of Marine and Environmental Research, University of Porto, \\ Edifício do Terminal de Cruzeiros do Porto de Leixões, Avenida General Norton de Matos, S/N, \\ 4450-208 Matosinhos, Portugal; vmvascon@fc.up.pt (V.V.); mrm@ess.ipp.pt (R.M.); pleao@ciimar.up.pt (P.L.) \\ 3 LAQV/REQUIMTE, Laboratory of Microbiology, Department of Biological Sciences, Faculty of Pharmacy, \\ University of Porto, Rua Jorge Viterbo Ferreira, 228, 4050-313 Porto, Portugal; \\ JoanaAlmeida15@gmail.com (J.A.); naircampos@gmail.com (N.N.) \\ 4 LAQV/REQUIMTE, Department of Chemistry and Biochemistry, Faculty of Sciences, University of Porto, \\ Rua do Campo Alegre 687, 4169-007 Porto, Portugal; idias@fc.up.pt \\ 5 Department of Biology, Faculty of Sciences, University of Porto, Rua do Campo Alegre, Edifício FC4, \\ 4169-007 Porto, Portugal \\ 6 Health and Environment Research Centre, School of Health, Polytechnic Institute of Porto, Rua Dr. António \\ Bernardino de Almeida, 400, 4200-072 Porto, Portugal \\ * Correspondence: lucilia.saraiva@ff.up.pt (L.S.); hcidade@ff.up.pt (H.C.); \\ Tel.: +351-220428584 (L.S.); +351-220428688 (H.C.) \\ + These authors contributed equally to this work.
}

Received: 10 March 2020; Accepted: 29 March 2020; Published: 30 March 2020

\begin{abstract}
Hierridin B (6), a methylated hydroquinone isolated from the marine picocyanobacterium Cyanobium sp. LEGE 06113, moderately inhibited the growth of colon adenocarcinoma HT-29 cells. Aiming to improve the potential antitumor activity of this natural product, the demethylated analogue, norhierridin B (10), as well as its structurally-related quinone (9), were synthesized and evaluated for their growth inhibitory effect on a panel of human tumor cell lines, including the triple-negative breast cancer (TNBC) cells MDA-MB-231, SKBR3, and MDA-MB-468. Norhierridin B (10) showed a potent growth inhibitory effect on all cancer cell lines. Moreover, the growth inhibitory effect of compound 10 on MDA-MB-231 cells was associated with cell cycle arrest and apoptosis. Norhierridin B (10) interfered with several p53 transcriptional targets, increasing p21, Bax, and MDM2, while decreasing Bcl-2 protein levels, which suggested the potential activation of a p53 pathway. Altogether, these results evidenced a great improvement of the antitumor activity of hydroquinone $\mathbf{1 0}$ when compared to 6 and its structurally-related quinone (9). Notably, hydroquinone 10 displayed a prominent growth inhibitory activity against TNBC cells, which are characterized by high therapeutic resistance.
\end{abstract}

Keywords: antitumor activity; hierridin B; hydroquinones; quinones 


\section{Introduction}

Quinones/hydroquinones constitute a family of metabolites, widespread in nature, with a wide range of biological activities, including cytotoxicity to cancer cells [1-3]. Particularly, alkyl 1,4-benzoquinones/hydroquinones with aliphatic side chains have been found attractive for their in vitro growth inhibitory effect against several human cancer cell lines [3,4] (Figure 1). Among these secondary metabolites, a wide array of bioactive compounds has been isolated from nature, namely from plant species and marine organisms. For instance, compounds 1-3 (Figure 1), isolated from Ardisia virens, exhibited a potent inhibitory effect against breast adenocarcinoma MCF-7, non-small cell lung cancer NCI-H460, and glioblastoma SF-268 cell lines (concentration that caused cell growth inhibition of $50 \%, \mathrm{GI}_{50}$, of 2.21-11.32 $\mu \mathrm{M}$ ) [4], with hydroquinone 3 being more potent than quinone 2. Toluquinol (4) (Figure 1), isolated from the marine fungus Penicillium sp. HL-85-ALS5-R004, is another example of a natural product with a promising in vitro growth inhibitory effect in breast adenocarcinoma MDA-MB-231 and glioblastoma U87-MG cells, as well as in promyelocytic leukemia HL-60, fibrosarcoma HT-1080, and colorectal adenocarcinoma HT-29 cell lines [5]. Interestingly, when comparing the in vitro growth inhibitory activity of quinones with structurally related hydroquinones, it seems that the presence of a hydroquinone could be more favorable for activity. In fact, the $\mathrm{IC}_{50}$ values obtained for quinone 2 are slightly higher than those of the structurally related hydroquinone 3 [4]. Moreover, toluquinone (5), a synthetic analogue of the marine hydroquinone toluquinol (4), showed lower in vitro growth inhibitory activity against a panel of cancer cell lines of breast adenocarcinoma (MDA-MB-231, $\mathrm{GI}_{50}$ of $3.4 \pm 0.1 \mu \mathrm{M}$ ), promyelocytic leukemia (HL-60, $\mathrm{GI}_{50}$ of $5.6 \pm 1.9 \mu \mathrm{M}$ ), glioblastoma (U87-MG, $\mathrm{GI}_{50}$ of $\left.8.8 \pm 0.9 \mu \mathrm{M}\right)$, fibrosarcoma (HT-1080, $\mathrm{GI}_{50}$ of $\left.4.9 \pm 1.7 \mu \mathrm{M}\right)$, and colorectal adenocarcinoma (HT-29, $\mathrm{GI}_{50}$ of $9.5 \pm 0.5 \mu \mathrm{M}$ ) than toluquinol (5) (MDA-MB-231: $\mathrm{GI}_{50}$ of $2.3 \pm 0.8 \mu \mathrm{M}, \mathrm{HL}-60: \mathrm{GI}_{50}$ of $1.7 \pm 0.5 \mu \mathrm{M}, \mathrm{U} 87-\mathrm{MG}: \mathrm{GI}_{50}$ of $5.6 \pm 1.5 \mu \mathrm{M}, \mathrm{HT}-1080: \mathrm{GI}_{50}$ of $1.4 \pm 0.6 \mu \mathrm{M}$ and HT-29: $\mathrm{GI}_{50}$ of $4.1 \pm 0.4 \mu \mathrm{M}$ ), further supporting that the presence of a hydroquinone is favorable for the antitumor activity [5].<smiles>COC1=CC(=O)C=C(C[C@@H](CCC(C)=O)OC(C)=O)C1=O</smiles>

1: $n=11$

2: $n=9$<smiles>CCCCC(Cc1cc(O)cc(OC)c1O)OC(C)=O</smiles>

3<smiles>Cc1cc(O)ccc1O</smiles><smiles>CC1=CC(=O)C=CC1=O</smiles><smiles>CCCCCCCCCCCCCCCc1cc(OC)cc(OC)c1O</smiles>

Toluquinol (4) Toluquinone (5)

Hierridin B (6)

Figure 1. Structure of quinones and hydroquinones with cytotoxic activity. 
Recently, following the search for new bioactive secondary metabolites from marine cyanobacteria, we identified hierridin B (6), a methylated hydroquinone derivative with a C15 aliphatic chain isolated from the marine picocyanobacterium Cyanobium sp. LEGE 06,113 [6]. Compound 6 moderately inhibited the growth of colon adenocarcinoma HT-29 cell line, with a GI $_{50}$ of $100.2 \mu \mathrm{M}$ [6]. In order to obtain new structurally-related quinone/hydroquinone with improved antiproliferative activity in cancer cell lines, the demethylated hierridin B derivative, norhierridin B (10), as well as structurally-related quinone 9 and compound 6, were synthesized and evaluated for their growth inhibitory effect in a panel of human tumor cell lines.

\section{Results and Discussion}

\subsection{Synthesis}

Norhierridin B (10) was synthesized according to the strategy illustrated on Scheme 1. In order to explore the growth inhibitory effect of demethylated hierridin B (6) derivative (10), as well as of its 1,4-benzoquinone analogue (9), a synthetic pathway was planned in order to obtain this analogue as an intermediate. Firstly, Grignard addition to $o$-vanilin using tetradecyl magnesium bromide in situ prepared from $\mathrm{Mg}$ turnings and tetradecyl bromide in dry diethyl ether afforded the corresponding carbinol 7 with $14 \%$ yield. The reduction of $\alpha$-hydroxy group was firstly attempted by hydrogenolysis catalyzed by palladium on carbon (Pd/C) using sulfuric acid as hydrogen donor, as reported by González et al. [7]. Nevertheless, as no reaction occurred, a similar reaction with Pd/C using formic acid as hydrogen donor was conducted, affording the reduced derivative 8 with 93\% yield. The high yield obtained reinforces the advantage of using palladium catalysts and formic acid in the catalytic transfer hydrogenolysis of benzylic alcohols, as described previously [8-10]. Phenol 8 was oxidized by streaming oxygen into a solution of DMF containing salcomine as catalyst, affording the 1,4-benzoquinone 9 with good yield (82\%). The reduction of quinone 9 using $\mathrm{Na}_{2} \mathrm{~S}_{2} \mathrm{O}_{4}$ as a reducing agent afforded norhierridin B (10) with $87 \%$ yield.<smiles>COc1cccc(C=O)c1O</smiles>
$o$-Vanilin<smiles>CCCCCCCC(O)c1cccc(OC)c1O</smiles>
7

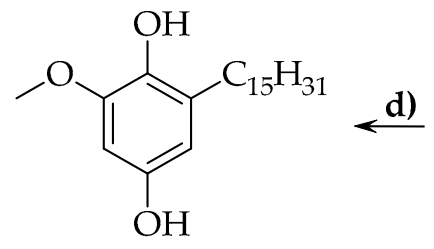

Norhierridin B (10)

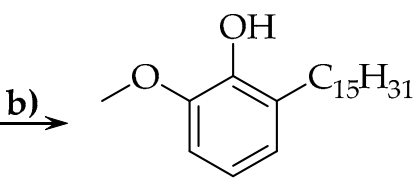

8<smiles>C1CCOC1</smiles>

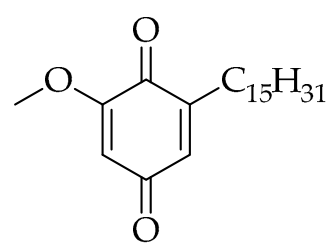

9

Scheme 1. Synthesis of norhierridin B (10). (a) $\mathrm{C}_{14} \mathrm{H}_{29} \mathrm{Br}, \mathrm{Mg}, \mathrm{Et}_{2} \mathrm{O}, 35^{\circ} \mathrm{C}, 2 \mathrm{~h}$; (b) $\mathrm{HCOOH}, \mathrm{Pd} / \mathrm{C}$, $\mathrm{EtOH} / \mathrm{H}_{2} \mathrm{O}, 80^{\circ} \mathrm{C}, 4$ h; (c) $\mathrm{O}_{2}$, salcomine, DMF, r. t., 24 h; (d) $\mathrm{Na}_{2} \mathrm{~S}_{2} \mathrm{O}_{4}, \mathrm{CHCl}_{3} / \mathrm{H}_{2} \mathrm{O}$, r.t., 10 min.

The structure elucidation of the new hydroquinone 10 was established by NMR and HRMS (Figures S4 and S5). The newly synthesized quinone 9, as well as intermediates 7 and 8, were characterized by NMR techniques, and data were in accordance with that previously reported [11,12]. ${ }^{13} \mathrm{C}-\mathrm{NMR}$ assignments were determined by 2D heteronuclear single quantum correlation (HSQC) and heteronuclear multiple bond correlation (HMBC) experiments. Hierridin B (6) was synthesized and characterized as described previously [13]. 


\subsection{Biological Activity}

Tumor Cell Growth Inhibitory Activity Evaluation

The growth inhibitory effect of norhierridin B (10), as well as of its structurally related 1,4-benzoquinone (9), intermediates 7 and 8, and hierridin B (6), was evaluated in a panel of distinct human cancer cell lines of melanoma (A375), hepatocarcinoma (Huh-7), colon (HCT116), and triple-negative breast cancer (TNBC) (MDA-MB-231, SKBR3, and MDA-MB-468), using the sulforhodamine (SRB) assay. A dose-response curve was obtained for each compound in the distinct cell lines and the concentration that induced half of growth inhibition $\left(\mathrm{GI}_{50}\right)$ was determined after $48 \mathrm{~h}$ treatment (Table 1).

Table 1. Effect of compounds $\mathbf{6 - 1 0}$ on the growth of human cancer cell lines.

\begin{tabular}{ccccccc}
\hline & \multicolumn{7}{c}{$\mathbf{G I}_{\mathbf{5 0}}(\boldsymbol{\mu M})$} \\
\cline { 2 - 7 } Cell line/Compound & $\mathbf{6}$ & $\mathbf{7}$ & $\mathbf{8}$ & $\mathbf{9}$ & $\mathbf{1 0}$ & Etoposide \\
\hline MDA-MB-231 & $>50$ & $9.65 \pm 0.15$ & $28.8 \pm 3.3$ & $5.83 \pm 1.10$ & $0.61 \pm 0.07$ & $5.06 \pm 1.24$ \\
SKBR3 & $>50$ & $14.0 \pm 0.0$ & $>50$ & $4.35 \pm 0.15$ & $0.77 \pm 0.06$ & $4.77 \pm 1.09$ \\
MDA-MB-468 & $>50$ & $7.85 \pm 2.15$ & $31.00 \pm 7.00$ & $6.65 \pm 0.90$ & $0.68 \pm 0.13$ & $2.14 \pm 0.36$ \\
A375 & $>50$ & $16.0 \pm 1.7$ & $32.9 \pm 4.2$ & $20.6 \pm 1.9$ & $2.0 \pm 0.4$ & $0.9 \pm 0.07$ \\
Huh-7 & $>50$ & $27.5 \pm 0.5$ & $>50$ & $2.95 \pm 0.15$ & $0.61 \pm 0.03$ & $3.43 \pm 0.85$ \\
HCT116 & $>50$ & $26.5 \pm 0.5$ & $26.0 \pm 3.2$ & $29.6 \pm 0.5$ & $3.2 \pm 0.6$ & $0.67 \pm 0.05$ \\
\hline
\end{tabular}

$\mathrm{GI}_{50}$ was determined by sulforhodamine (SRB) assay after $48 \mathrm{~h}$ treatment (growth obtained with vehicle was set as

$100 \%$ ). Data are mean \pm SEM of two to five independent experiments. Etoposide was used as positive control.

The obtained $\mathrm{GI}_{50}$ values revealed that all newly synthesized compounds $(\mathbf{7}, \mathbf{8}, \mathbf{9}$, and 10$)$ had a much higher growth inhibitory activity than hierridin B (6) in all cancer cell lines (Table 1). Norhierridin B (10) was found to be the most effective against all cancer cell lines, exhibiting much lower $\mathrm{GI}_{50}$ values than its methyl derivative hierridin B (6). These results suggest that the presence of two $p$-hydroxy groups in the hydroquinone scaffold are important for the in vitro growth inhibitory activity. In addition, norhierridin B (10) displayed higher activity than its structurally related quinone (9), reinforcing that the presence of a hydroquinone scaffold is more favorable for the activity than a quinone scaffold, as already reported by Chang et al. [4] and Cheng-Sánchez et al. [5] for other structurally related hydroquinones/quinones.

Interestingly, norhierridin $B(\mathbf{1 0})$ exhibited a prominent growth inhibitory activity against TNBC cells MDA-MB-231, SKBR3, and MDA-MB-468, which are well-known by their high therapeutic resistance [14]. Moreover, an evident reduction of the norhierridin B (10) antiproliferative effect could be observed by SRB assay in non-tumorigenic MCF10A breast epithelial cell lines (10.36 $\pm 1.96 \mu \mathrm{M}$, three independent experiments). In fact, supporting the marked antiproliferative effect of compound 10 on MDA-MB-231 cells, observed by SRB assay (Figure 2A), a pronounced inhibition of the growth of these cancer cells was also observed at $2 \mu \mathrm{M}$ by colony formation assay (Figure 2B,C). 
A

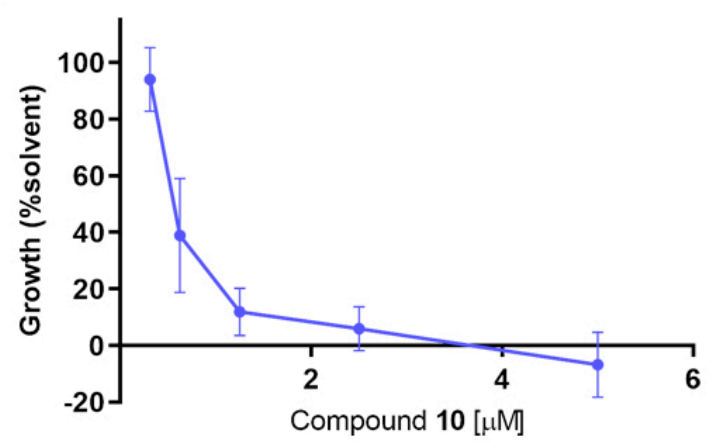

B
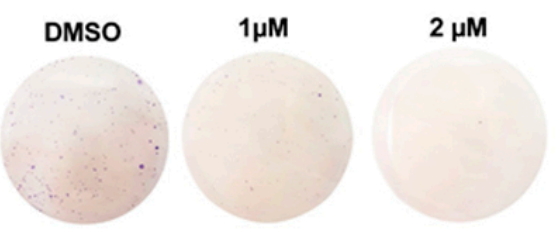

C

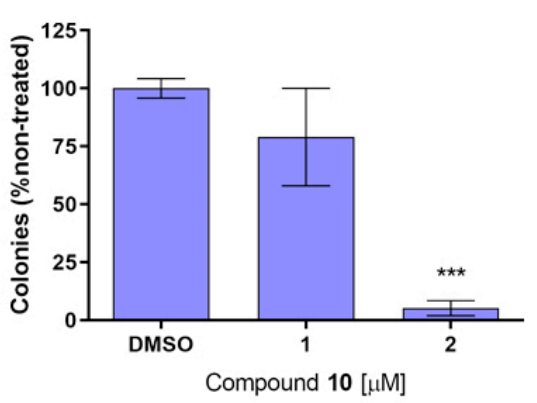

Figure 2. Norhierridin B (10) inhibits the growth of breast adenocarcinoma MDA-MB-231 cells. (A) Dose-response curve for the growth of MDA-MB-231 cells treated with compound $\mathbf{1 0}$ for $48 \mathrm{~h}$, determined by SRB assay; data are mean \pm SEM of four independent experiments; growth obtained with vehicle was set as 100\%. (B, C) Colony formation assay for MDA-MB-231 cells treated with compound $\mathbf{1 0}$ for nine days: (B) images correspond to a representative experiment of two; (C) graph represents mean \pm SEM of two independent experiments; values significantly different from DMSO: *** $p<0.001$, unpaired Student's $t$-test.

It was also verified that the growth inhibitory effect of compound 10 on MDA-MB-231 cells was associated with the induction of apoptosis and cell cycle arrest. In fact, the $1 \mu \mathrm{M}$ concentration of compound 10 caused a significant G0/G1-phase cell cycle arrest (Figure 3A), which was consistent with the visible increase of p21 protein expression levels observed by western blot analysis (Figure 3C). Moreover, by cell viability assay, 1 and $2 \mu \mathrm{M}$ of compound 10 significantly increased the percentage of dead cells (Figure 3B), which was associated with an increase of the pro-apoptotic protein Bax and a decrease of the anti-apoptotic protein Bcl-2 (Figure 3C). It is interesting to note that compound 10 interfered with the protein expression levels of several p53 transcriptional targets, such as p21, Bax, Bcl-2, and MDM2 (Figure 3C), suggesting the activation of a p53 pathway in MDA-MB-231 cells. Collectively, these results indicate that compound $\mathbf{1 0}$ is very effective against TNBC cells. The poor prognosis and limited therapeutic options commonly observed with this type of cancer [14] render compound $\mathbf{1 0}$ worthy of further studies to confirm its promising application as potential anticancer agent, particularly against TNBC. 
A

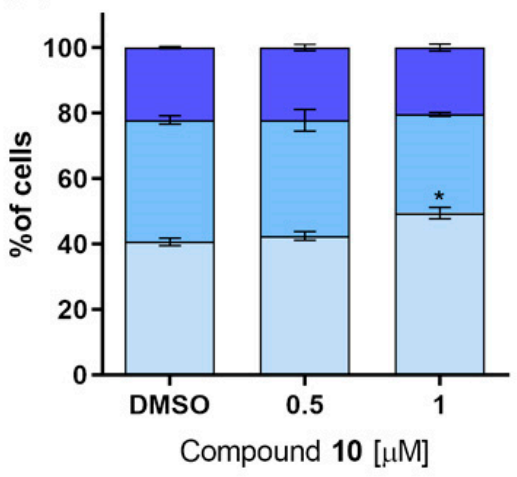

B

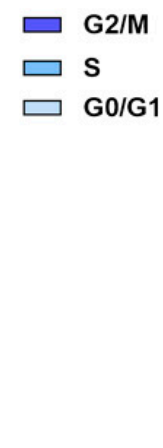

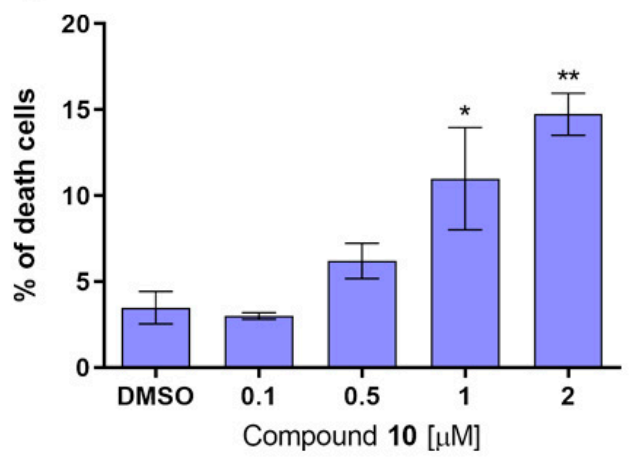

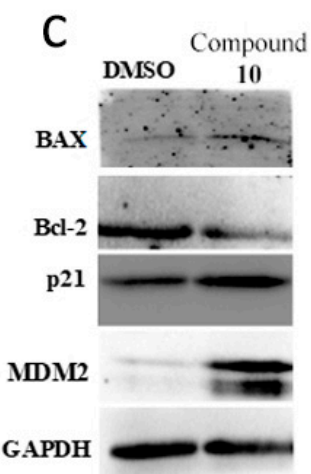

Figure 3. Norhierridin B (10) induces cell cycle arrest and death and interferes with p53 transcriptional targets. (A) Cell cycle progression was analyzed after $48 \mathrm{~h}$ treatment with compound 10 in MDA-MB-231 cells; data are mean \pm SEM of three independent experiments; values significantly different from DMSO: * $p<0.05$, unpaired Student's $t$-test. (B) Percentage of death cells determined by trypan blue assay for MDA-MB-231 cells treated with compound $\mathbf{1 0}$ for $48 \mathrm{~h}$; data are mean \pm SEM of three independent experiments; values significantly different from DMSO: ${ }^{*} p<0.05,{ }^{* *} p<0.001$, unpaired Student's $t$-test. (C) Protein expression levels of p53 targets in MDA-MB-231 cells was analyzed by western blot after $48 \mathrm{~h}$ treatment with $2 \mu \mathrm{M}$ compound 10. Immunoblots are representative of three independent experiments; GAPDH was used as loading control.

\section{Material and Methods}

\subsection{Synthesis}

All reactions were monitored by thin-layer chromatography (TLC). Purifications of compounds were carried out by flash chromatography using Macherey-Nagel silica gel $60(0.04-0.063 \mathrm{~mm})$. Melting points were obtained in a Köfler microscope (Wagner and Munz, Munich, Germany) and are uncorrected. ${ }^{1} \mathrm{H}-\mathrm{NMR}$ and ${ }^{13} \mathrm{C}-\mathrm{NMR}$ spectra were taken in $\mathrm{CDCl}_{3}$ at room temperature, on a Bruker Avance 300 instrument $\left(300.13 \mathrm{MHz}\right.$ for ${ }^{1} \mathrm{H}$ and $75.47 \mathrm{MHz}$ for ${ }^{13} \mathrm{C}$, Bruker Biosciences Corporation, Billerica, MA, USA). Chemical shifts are expressed in ppm values relative to tetramethylsilane (TMS) used as an internal reference; ${ }^{13} \mathrm{C}-\mathrm{NMR}$ assignments were made by 2D (HSQC and HMBC) NMR experiments (long-range ${ }^{13} \mathrm{C}^{-1} \mathrm{H}$ coupling constants were optimized to $7 \mathrm{~Hz}$ ). HRMS mass spectrum was recorded as ESI (electrospray ionization) mode on a MicrOTOF spectrometer (Bruker Corporation, Billerica, MA, USA) at C.A.C.T.I.-University of Vigo, Spain. The commercially available reagents, and etoposide, were purchased from Sigma Aldrich Co. (Sintra, Portugal). Reagents and solvents were purified and dried according to the usual procedures described elsewhere [15]. Hierridin B (6) was synthesized as described previously [13]. The following materials were synthesized and purified by the described procedures. 


\subsubsection{Synthesis of 2-(1-hydroxypentadecyl)-6-methoxyphenol (7)}

Tetradecyl magnesium bromide was prepared from $\mathrm{Mg}$ turnings (2.01 g, 0.62 eq.) and tetradecyl bromide $(37 \mathrm{~g}, 0.13 \mathrm{~mol})$ in dry diethyl ether $(200 \mathrm{~mL})$ under a nitrogen atmosphere. $o$-Vanilin $(10 \mathrm{~g}$, $0.066 \mathrm{~mol}$ ) was gradually added, and the mixture was then refluxed for $2 \mathrm{~h}$, cooled, and poured into a saturated solution of $\mathrm{NH}_{4} \mathrm{Cl}(150 \mathrm{~mL})$. The layers were separated, and the aqueous phase thoroughly extracted with diethyl ether $(3 \times 200 \mathrm{~mL})$. The organic layer was washed with water, dried over anhydrous sodium sulfate, and evaporated under reduced pressure. The residue was purified by flash column chromatography (silica gel, $n$-hexane: ethyl acetate 8:2), affording $7(3.23 \mathrm{~g}, 14 \%)$ as a white solid.

2-(1-hydroxypentadecyl)-6-methoxyphenol (7): ${ }^{1} \mathrm{H}-\mathrm{NMR}\left(\mathrm{CDCl}_{3}, 300.13 \mathrm{MHz}\right): \delta=6.83-6.78(\mathrm{~m}, 3 \mathrm{H}$, $\mathrm{H}-3,-4,-5), 6.38(\mathrm{~s}, 1 \mathrm{H}, 1-\mathrm{OH}), 4.87\left(\mathrm{t}, J=6.0 \mathrm{~Hz}, 1 \mathrm{H}, \mathrm{H}-1^{\prime}\right), 3.88\left(\mathrm{~s}, 3 \mathrm{H}, 2-\mathrm{OCH}_{3}\right), 1.84-1.80\left(\mathrm{~m}, 2 \mathrm{H}, \mathrm{H}-2^{\prime}\right)$, $1.30-1.24\left(\mathrm{~m}, 24 \mathrm{H}, \mathrm{H}-3^{\prime}-14^{\prime}\right), 0.87\left(\mathrm{t}, J=6.5 \mathrm{~Hz}, 3 \mathrm{H}, \mathrm{H}-15^{\prime}\right) ;{ }^{13} \mathrm{C}-\mathrm{NMR}\left(\mathrm{CDCl}_{3}, 75.47 \mathrm{MHz}\right) \delta: 146.8$ (C-2), 143.1 (C-1), 129.8 (C-6), 119.6 (C-4), $119.2(\mathrm{C}-5), 109.7$ (C-3), $72.0\left(\mathrm{C}-1^{\prime}\right), 56.0\left(2-\mathrm{OCH}_{3}\right), 37.2\left(\mathrm{C}-2^{\prime}\right)$, 31.9-22.7 (C-2'-14'), $14.1\left(\mathrm{C}-15^{\prime}\right)$.

\subsubsection{Synthesis of 2-methoxy-6-pentadecylphenol (8)}

In a $250 \mathrm{~mL}$ two neck round-bottomed flask equipped with a water-cooled condenser and a magnetic stirrer, ethanol $(99 \mathrm{~mL})$, water $(20 \mathrm{~mL})$, compound $7(3.17 \mathrm{~g}, 9 \mathrm{mmol})$, formic acid $(0.68 \mathrm{~mL})$, and $\mathrm{Pd} / \mathrm{C} 10 \%(w / w)(1.259 \mathrm{~g})$ were added. The reactor was placed in a preheated oil bath and maintained at $80^{\circ} \mathrm{C}$ under atmospheric pressure for $4 \mathrm{~h}$. The reactor was allowed to cool down to r.t., and the ethanol was evaporated under reduced pressure. Water was added $(20 \mathrm{~mL})$, and the resulting solution was extracted with diethyl ether $(3 \times 100 \mathrm{~mL})$. The organic layer was dried over anhydrous sodium sulfate and evaporated under reduced pressure affording a solid, which was crystallized from diethyl ether: methanol affording the title compound $(8)(2,81 \mathrm{~g}, 93 \%)$ as a white solid.

2-methoxy-6-pentadecylphenol (8): ${ }^{1} \mathrm{H}-\mathrm{NMR}\left(\mathrm{CDCl}_{3}, 300.13 \mathrm{MHz}\right): \delta=6.80-6.70(\mathrm{~m}, 3 \mathrm{H}, \mathrm{H}-3,-4,-5), 5.66$ $(\mathrm{s}, 1 \mathrm{H}, 1-\mathrm{OH}), 3.87\left(\mathrm{~s}, 3 \mathrm{H}, 2-\mathrm{OCH}_{3}\right), 2.62\left(\mathrm{t}, J=7.6 \mathrm{~Hz}, 1 \mathrm{H}, \mathrm{H}-1^{\prime}\right), 1.65-1.57\left(\mathrm{~m}, 2 \mathrm{H}, \mathrm{H}-2^{\prime}\right), 1.27-1.25$ $\left(\mathrm{m}, 24 \mathrm{H}, \mathrm{H}-3^{\prime}-14^{\prime}\right), 0.87\left(\mathrm{t}, J=6.5 \mathrm{~Hz}, 3 \mathrm{H}, \mathrm{H}-15^{\prime}\right) ;{ }^{13} \mathrm{C}-\mathrm{NMR}\left(\mathrm{CDCl}_{3}, 75.47 \mathrm{MHz}\right) \delta: 146.3(\mathrm{C}-2), 143.4$ (C-1), 128.8 (C-6), $122.3(\mathrm{C}-5), 119.1(\mathrm{C}-4), 108.1(\mathrm{C}-3), 55.9\left(2-\mathrm{OCH}_{3}\right), 31.9\left(\mathrm{C}-2^{\prime}\right), 30.9\left(\mathrm{C}-1^{\prime}\right), 37.2\left(\mathrm{C}-2^{\prime}\right)$, 29.8-22.7 (C-2'-14'), $14.1\left(\mathrm{C}-15^{\prime}\right)$.

\subsubsection{Synthesis of 2-methoxy-6-pentadecyl-1,4-benzoquinone (9)}

A solution of $8(2.17 \mathrm{~g}, 6 \mathrm{mmol})$ in DMF was bubbled with $\mathrm{O}_{2}$ for $5 \mathrm{~min}$ and, after the $\mathrm{O}_{2}$ atmosphere, was kept over the reaction vessel. Salcomine was added $(380 \mathrm{mg}, 1.17 \mathrm{mmol})$, and the reaction was stirred at r.t. for $24 \mathrm{~h}$. After, diethyl ether $(20 \mathrm{~mL})$ was added, and the organic layer was washed with $0.1 \mathrm{M} \mathrm{HCl}(2 \times 10 \mathrm{~mL})$, water $(10 \mathrm{~mL})$ and brine $(10 \mathrm{~mL})$. The organic layer was dried over anhydrous sodium sulfate, and the solvent evaporated. The obtained solid was purified by crystallization from petroleum ether $40-60{ }^{\circ} \mathrm{C}$, affording the title compound (9) $(1.85 \mathrm{~g}, 82 \%)$ as a yellow solid.

2-methoxy-6-pentadecyl-1,4-benzoquinone (9): ${ }^{1} \mathrm{H}-\mathrm{NMR}\left(\mathrm{CDCl}_{3}, 300.13 \mathrm{MHz}\right): \delta=6.49-6.47(\mathrm{~m}, 1 \mathrm{H}, \mathrm{H}-5)$, $5.87(\mathrm{~d}, J=2.4 \mathrm{~Hz}, 1 \mathrm{H}, \mathrm{H}-3), 3.82\left(\mathrm{~s}, 3 \mathrm{H}, 2-\mathrm{OCH}_{3}\right), 2.44\left(\mathrm{ddd}, \mathrm{J}=7.5 \mathrm{~Hz}, 1.4,2 \mathrm{H}, \mathrm{H}-\mathrm{1}^{\prime}\right), 1.54-1.45(\mathrm{~m}, 2 \mathrm{H}$, H-2' $), 1.30-1.25$ (m, 24 H, H-3'-14' $), 0.88\left(\mathrm{t}, J=6.5,3 \mathrm{H}, \mathrm{H}-15^{\prime}\right) ;{ }^{13} \mathrm{C}-\mathrm{NMR}\left(\mathrm{CDCl}_{3}, 75.47 \mathrm{MHz}\right) \delta: 187.7$ (4-CO), 182.2 (1-CO), 158.9 (C-2), 147.6 (C-6), 132.9 (C-5), 107.1 (C-3), $55.3\left(2-\mathrm{OCH}_{3}\right), 28.7\left(\mathrm{C}-1^{\prime}\right), 27.7$ (C-2'), 29.8-22.7 (C-2'-14'), 14.1 (C-15').

\subsubsection{Synthesis of 2-methoxy-6-pentadecylbenzene-1,4-diol (10).}

Compound $9(1.86 \mathrm{~g}, 0.005 \mathrm{~mol})$ was dissolved in chloroform and added to a $10 \%$ solution of $\mathrm{Na}_{2} \mathrm{~S}_{2} \mathrm{O}_{4}$ in hot water. The mixture was shaken for $10 \mathrm{~min}$, and, after the aqueous layer was drawn off, the chloroform was shaken with brine, dried over anhydrous sodium sulfate, and the solvent 
evaporated under reduced pressure. The residue was purified by crystallization from $n$-hexane, affording the title compound (10) $(1.31 \mathrm{~g}, 87 \%)$ as a white solid.

2-methoxy-6-pentadecylbenzene-1,4-diol (10): m.p. (n-hexane) $=92-94{ }^{\circ} \mathrm{C} ;{ }^{1} \mathrm{H}-\mathrm{NMR}\left(\mathrm{CDCl}_{3}, 300.13 \mathrm{MHz}\right)$ : ${ }^{1} \mathrm{H}-\mathrm{NMR}\left(\mathrm{CDCl}_{3}, 300.13 \mathrm{MHz}\right): \delta 6.31(\mathrm{~d}, J=2.8 \mathrm{~Hz}, 1 \mathrm{H}, \mathrm{H}-3), 6.20(\mathrm{~d}, J=2.8 \mathrm{~Hz}, 1 \mathrm{H}, \mathrm{H}-5), 3.84(\mathrm{~s}, 3 \mathrm{H}$, 2- $\left.\mathrm{OCH}_{3}\right), 2.56\left(\mathrm{t}, J=7.5 \mathrm{~Hz}, 2 \mathrm{H}, \mathrm{H}-1^{\prime}\right), 1.60-1.52\left(\mathrm{~m}, 2 \mathrm{H}, \mathrm{H}-2^{\prime}\right), 1.30-1.25\left(\mathrm{~m}, 24 \mathrm{H}, \mathrm{H}-3^{\prime}-14^{\prime}\right), 0.87(\mathrm{t}, J=$ $\left.6.5 \mathrm{~Hz}, 3 \mathrm{H}, \mathrm{H}-15^{\prime}\right) ;{ }^{13} \mathrm{C}-\mathrm{NMR}\left(\mathrm{CDCl}_{3}, 75.47 \mathrm{MHz}\right): \delta 148.3$ (C-2), 147.6 (C-4), 137.3 (C-1), 107.9 (C-5), $97.0(\mathrm{C}-3), 56.3\left(2-\mathrm{OCH}_{3}\right), 31.9\left(\mathrm{C}-2^{\prime}\right), 30.9\left(\mathrm{C}-1^{\prime}\right), 29.8-22.7\left(\mathrm{C}-2^{\prime}-14^{\prime}\right), 14.1$ (C-15'); ESI-TOF-HRMS (+) $m / z$ : Anal. Calc. for $\mathrm{C}_{24} \mathrm{H}_{37} \mathrm{O}_{3}\left(\mathrm{M}+\mathrm{H}^{+}\right)$: 349.27320; found: 349.27372 .

\subsection{Biological Activity}

\subsubsection{Human Cell Lines and Growth Conditions}

Human breast adenocarcinoma MDA-MB-231, SK-BR-3, MDA-MB-468, melanoma A375, hepatocellular carcinoma Huh-7, colorectal carcinoma HCT116, and non-tumorigenic breast epithelial MCF10A cell lines were purchased from ATCC (Rockville, MD, USA). Cancer cells were cultured in RPMI-1640 medium with UltraGlutamine (Biowest, VWR, Carnaxide, Portugal) with $10 \%$ fetal bovine serum (FBS; Gibco, Alfagene, Lisboa, Portugal). MCF10a cells were cultured in DMEM:F12 with 10\% FBS or MEGM SingleQuot Kit Suppl. \& Growth Factors, respectively (Lonza, VWR, Carnaxide, Portugal). Cells were maintained at $37^{\circ} \mathrm{C}$ in a humidified atmosphere of $5 \% \mathrm{CO}$. Mycoplasma detection was routinely performed using the MycoAlert ${ }^{\mathrm{TM}}$ PLUS kit (Lonza).

\subsubsection{Sulforhodamine B (SRB) Assay}

Human cell lines were incubated in 96-well plates at a final density of $5.0 \times 10^{3}$ cells/well (for A375, Huh-7 and HCT116 cells), $1.0 \times 10^{4}$ cells/well (for MDA-MB-231 and MCF10A) and 7.5 $\times 10^{3}$ cells/well (for SKBR3 and MDA-MB-468 cells) for $24 \mathrm{~h}$. Cells were then treated with serial dilutions $(0.313 \mu \mathrm{M}$ to $50 \mu \mathrm{M})$ of compounds for $48 \mathrm{~h}$. The effect of these compounds on in vitro growth was assessed using the SRB assay. Cells were fixated with $12.5 \%$ TCA for $1 \mathrm{~h}$ at $4{ }^{\circ} \mathrm{C}$. Plates were then stained with $0.25 \%$ SRB (Sigma-Aldrich, Sintra, Portugal) and washed with $1 \%$ acetic acid. The bound dye was solubilized in $0.12 \%$ Tris Base and the absorbance was measured at $510 \mathrm{~nm}$ in a microplate reader (Biotek Instruments Inc., Synergy, MX, USA). The maximum concentration of solvent used in this assay $\left(1 \%\right.$ DMSO) was included as control. The $\mathrm{GI}_{50}$ was then determined.

\subsubsection{Colony Formation Assay}

MDA-MB-231 cells were seeded in 6-well plates at a density of $5.0 \times 10^{2}$ cells/well, followed by incubation with hydroquinone 10 for 9 days. Formed colonies were fixed with $10 \% \mathrm{MeOH}$ and $10 \%$ $\mathrm{AcOH}$ for $10 \mathrm{~min}$, and then they were stained with $0.5 \%$ crystal violet (Sigma-Aldrich, Sintra, Portugal) in $1: 1 \mathrm{MeOH} / \mathrm{H}_{2} \mathrm{O}$ for $15 \mathrm{~min}$. Colonies containing more than 20 cells were counted.

\subsubsection{Cell Viability Assay}

The percentage of viable cells was determined using the Trypan Blue Assay. Briefly, MDA-MB-231 cells were seeded in 24-well plates at $6 \times 10^{4}$ cells/well for $24 \mathrm{~h}$, followed by treatment with hydroquinone 10 for $24 \mathrm{~h}$. Cells were then harvested and resuspended in $0.4 \%$ Trypan Blue (Sigma-Aldrich, Sintra, Portugal), and the number of viable/dead cells were counted using a Leica light optical microscope (Wetzlar). Vehicle ( $0.25 \%$ DMSO) was included as a control.

\subsubsection{Cell Cycle Analysis}

Cell cycle and apoptosis were analyzed as described [16]. Briefly, MDA-MB-231 cells were incubated in 6-well plates at a density of $2.25 \times 10^{5}$ cells/well for $24 \mathrm{~h}$. Cells were then treated with hydroquinone 10 or DMSO only for $48 \mathrm{~h}$. For cell cycle analysis, cells were stained with propidium 
iodide (PI; Sigma-Aldrich, Sintra, Portugal); cell cycle scattering was analyzed by flow cytometry and cell cycle phases were quantified using FlowJoX v10.0.7 (Treestar, Woodburn, OR, USA).

\subsubsection{Western Blot Analysis}

MDA-MB-231 cells were seeded in 6-well plates at a density of $2.25 \times 10^{5}$ cells/well for $24 \mathrm{~h}$, followed by treatment with $2 \mu \mathrm{M}$ hydroquinone 10. Protein extracts were quantified using the Bradford reagent (Sigma-Aldrich, Sintra, Portugal). Proteins were run in SDS-PAGE and transferred to a Whatman nitrocellulose membrane from Protan (VWR, Carnaxide, Portugal). After blocking, proteins were identified using a mouse monoclonal anti-anti-MDM2 (D-12), anti-Bax (2D2), anti-Bcl-2 (C-2), or a rabbit polyclonal anti-p21 (C-19), followed by an anti-mouse or anti-rabbit horseradish-peroxidase (HRP)-conjugated secondary antibody. For the loading control, a mouse monoclonal anti-GAPDH (6C5) was used. The signal was detected with the ECL Amersham kit from GE Healthcare (VWR, Carnaxide, Portugal). The ChemiDoc ${ }^{\mathrm{TM}}$ XRS Imaging System from Bio-Rad Laboratories (Amadora, Portugal) for Microsoft Windows 10 was used for detection.

\subsubsection{Statistical analysis}

Data were statistically analyzed using GraphPad Prism version 7.0. Different statistical tests were used accordingly to the dataset; $p$ values $<0.05$ or $<0.001$ were considered statistically significant.

\section{Conclusions}

In this work, the demethylated derivative of hierridin B (6) and norhieridin B (10), as well as its structurally related quinone (9), were synthesized for the first time. The study of their effect on the in vitro cell growth inhibition of human tumor cell lines revealed that norhierridin B (10) was the most effective in all cancer cell lines, exhibiting much lower $\mathrm{IC}_{50}$ values than its structurally related quinone (9) and hierridin B (6). Particularly, hydroquinone 10 exhibited a noticeable growth inhibitory activity against the TNBC cells MDA-MB-231, SKBR3, and MDA-MB-468, with these being effects associated with cell cycle arrest; increase of $\mathrm{p} 21$ and Bax; and decrease of $\mathrm{Bcl}-2$ protein expression levels. The interference of hydroquinone 10 in MDA-MB-231 cells with the protein expression levels of the p53 transcriptional targets p21, Bax, Bcl-2, and MDM2 suggests the activation of a p53 pathway, which should be further explored in the future. Altogether, these results indicate that hydroquinone $\mathbf{1 0}$ could be an effective cytotoxic agent against TNBC cells, which should be further explored in the future.

Supplementary Materials: The following are available online, Figure S1: ${ }^{1} \mathrm{H}$ and ${ }^{13} \mathrm{C}-\mathrm{NMR}$ of compound 7, Figure S2: ${ }^{1} \mathrm{H}$ and ${ }^{13} \mathrm{C}-\mathrm{NMR}$ of compound 8, Figure S3: ${ }^{1} \mathrm{H}$ and ${ }^{13} \mathrm{C}-\mathrm{NMR}$ of compound 9, Figure $\mathrm{S} 4:{ }^{1} \mathrm{H}$ and ${ }^{13} \mathrm{C}-\mathrm{NMR}$ of compound 10, Figure S5: HRMS of compound 10.

Author Contributions: V.V., R.M., P.L., M.P., and H.C. conceptualized the study. H.C. planned all aspects of the study. J.M. and P.B. performed the synthesis, purification, and structure elucidation of compounds 7-10. I.E.S.-D. performed the synthesized of compound 6. J.M. wrote the manuscript. N.N. and J.A. performed the tumor cell growth assays. H.C. and M.P. designed the experimental work concerning the synthesis. L.S. designed the experimental work concerning the antitumor activity. V.V., R.M, P.L., L.S., and H.C. revised the manuscript. All authors agreed to the final version of the manuscript.

Funding: This research was supported by national funds through FCT-Foundation for Science and Technology within the scope of UIDB/04423/2020, UIDP/04423/2020 and UIDB/50006/2020. Joana Moreira acknowledges her grant (SFRH/BD/135852/2018).

Acknowledgments: The authors thank Sara Cravo for all the technical and scientific support.

Conflicts of Interest: The authors declare no conflict of interest. The funders had no role in the design of the study; in the collection, analyses, or interpretation of data; in the writing of the manuscript, or in the decision to publish the results. 


\section{References}

1. García, P.; Hernández, Á.; San Feliciano, A.; Castro, M. Bioactive Prenyl-and Terpenyl-Quinones/Hydroquinones of Marine Origin. Mar. Drugs. 2018, 16, 292. [CrossRef] [PubMed]

2. Asche, C. Antitumour quinones. Mini Rev. Med. Chem. 2005, 5, 449-467. [CrossRef] [PubMed]

3. Sunassee, S.N.; Davies-Coleman, M.T. Cytotoxic and antioxidant marine prenylated quinones and hydroquinones. Nat. Prod. Rep. 2012, 29, 513-535. [CrossRef] [PubMed]

4. $\quad$ Chang, H.-S.; Lin, Y.-J.; Lee, S.-J.; Yang, C.-W.; Lin, W.-Y.; Tsai, I.-L.; Chen, I.-S. Cytotoxic alkyl benzoquinones and alkyl phenols from Ardisia virens. Phytochemistry. 2009, 70, 2064-2071. [CrossRef] [PubMed]

5. Cheng-Sánchez, I.; Torres-Vargas, J.A.; Martínez-Poveda, B.; Guerrero-Vásquez, G.A.; Medina, M.Á.; Sarabia, F.; Quesada, A.R. Synthesis and Antitumor Activity Evaluation of Compounds Based on Toluquinol. Mar. Drugs. 2019, 17, 492. [CrossRef] [PubMed]

6. Leao, P.N.; Costa, M.; Ramos, V.; Pereira, A.R.; Fernandes, V.C.; Domingues, V.F.; Gerwick, W.H.; Vasconcelos, V.M.; Martins, R. Antitumor activity of hierridin B, a cyanobacterial secondary metabolite found in both filamentous and unicellular marine strains. PloS ONE. 2013, 8, e69562. [CrossRef] [PubMed]

7. Gonzalez, A.G.; Barrera, J.B.; Perez, E.M.R. Synthesis of hierridin, a phenol from the lichen, Ramalina hierrensis. Phytochemistry. 1992, 31, 1436-1439. [CrossRef]

8. Liu, X.; Lu, G.; Guo, Y.; Guo, Y.; Wang, Y.; Wang, X. Catalytic transfer hydrogenolysis of 2-phenyl-2-propanol over palladium supported on activated carbon. J. Mol. Catal. A: Chem. 2006, 252, 176-180. [CrossRef]

9. Feng, J.; Yang, C.; Zhang, D.; Wang, J.; Fu, H.; Chen, H.; Li, X. Catalytic transfer hydrogenolysis of

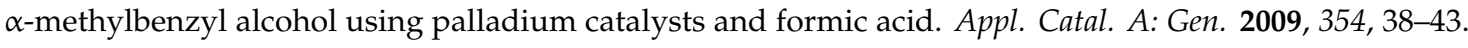
[CrossRef]

10. Sawadjoon, S.; Lundstedt, A.; Samec, J.S. Pd-catalyzed transfer hydrogenolysis of primary, secondary, and tertiary benzylic alcohols by formic acid: A mechanistic study. Acs Catal. 2013, 3, 635-642. [CrossRef]

11. König, W.A.; Faasch, H.; Heitsch, H.; Colberg, C.; Hausen, B.M. Synthese von seitenkettenmodifizierten Analogen des Allergens Primin/Synthesis of Side-Chain-Modified Analogues of the Allergen Primin. Z. Naturforsch. B. 1993, 48, 387-393. [CrossRef]

12. Davis, C.J.; Hurst, T.E.; Jacob, A.M.; Moody, C.J. Microwave-mediated Claisen rearrangement followed by phenol oxidation: A simple route to naturally occurring 1, 4-benzoquinones. The first syntheses of verapliquinones A and B and panicein A. J. Org. Chem. 2005, 70, 4414-4422. [CrossRef] [PubMed]

13. Costa, M.; Sampaio-Dias, I.E.; Castelo-Branco, R.; Scharfenstein, H.; Rezende de Castro, R.; Silva, A.; Schneider, M.P.C.; Araújo, M.J.O.; Martins, R.R.; Domingues, V.F. Structure of Hierridin C, Synthesis of Hierridins B and C, and Evidence for Prevalent Alkylresorcinol Biosynthesis in Picocyanobacteria. J. Nat. Prod. 2019, 82, 393-402. [CrossRef] [PubMed]

14. Mehanna, J.; Haddad, F.G.; Eid, R.; Lambertini, M.; Kourie, H.R. Triple-negative breast cancer: Current perspective on the evolving therapeutic landscape. Int. J. Womens Health. 2019, 11, 431. [CrossRef] [PubMed]

15. Perrin, D.; Armarego, W. Purification of Organic Chemicals, 3nd ed.; Pergamon Press: Oxford, UK, 1988; Volume 3, p. 65.

16. Soares, J.; Espadinha, M.; Raimundo, L.; Ramos, H.; Gomes, A.S.; Gomes, S.; Loureiro, J.B.; Inga, A.; Reis, F.; Gomes, C. DIMP 53-1: A novel small-molecule dual inhibitor of p53-MDM 2/X interactions with multifunctional p53-dependent anticancer properties. Mol. Oncol. 2017, 11, 612-627. [CrossRef]

Sample Availability: Samples of the compounds 7, 9 and 10 are available from the authors. 